PROCEEDINGS OF THE

AMERICAN MATHEMATICAL SOCIETY

Volume 128, Number 5, Pages 1459-1466

S 0002-9939(99)05533-1

Article electronically published on December 8, 1999

\title{
CURVATURE RESTRICTIONS ON CONVEX, TIMELIKE SURFACES IN MINKOWSKI 3-SPACE
}

\author{
SENCHUN LIN
}

(Communicated by Peter Li)

\begin{abstract}
Suppose that $K$ and $H$ are Minkowski Gauss curvature and Minkowski mean curvature respectively on a timelike surface $S$ that is $C^{2}$ immersed in Minkowski 3-space $E_{1}^{3}$. Suppose also that $0 \not \equiv K<0$ and that $S$ is complete as a surface in the underlying Euclidean 3 -space $E^{3}$. It is shown that neither $K$ nor $H$ can be bounded away from zero on such a surface $S$.
\end{abstract}

\section{INTRODUCTION}

The global behavior of timelike surfaces in Minkowski 3 -space $E_{1}^{3}$ is not yet fully understood. In particular, the effects of many standard restrictions on mean curvature $H$ or Gauss curvature $K$ are still not known. In this paper, we study the implications of such restrictions on timelike surfaces in $E_{1}^{3}$ that are convex as surfaces in Euclidean 3-space $E^{3}$.

A surface $S$ is convex if it is the boundary $\partial C$ of a closed, convex set $C$ with non-empty interior in $E^{3}$. (The Euclidean geometry of such surfaces has been extensively studied.) If $0 \not \equiv K<0$ on a timelike surface $S$ in $E_{1}^{3}$ that is complete as a surface in $E^{3}$, then $S$ is convex as a surface in $E^{3}$. We exploit this fact to show that if $0 \not \equiv K<0$ on a timelike surface $S$ in $E_{1}^{3}$ that is complete as a surface in $E^{3}$, then neither $H$ nor $K$ can be bounded away from zero on $S$. As a corollary, we show that (up to a rigid motion of $E_{1}^{3}$ ) a timelike surface $S$ in $E_{1}^{3}$ must be a circular cylinder or a hyperbolic cylinder in case $S$ is complete as a surface in $E^{3}$ with $H \equiv$ constant $\neq 0$ and $K \leq 0$ on $S$.

Our result, as it applies to $K$, can be compared with the Bonnet-Hopf-Rinow theorem, which states that any complete surfaces $S$ in $E^{3}$ on which Euclidean Gauss curvature $K_{\epsilon} \geq$ constant $>0$ must be compact. The conclusions match, since there are no compact timelike (or spacelike) surfaces in $E_{1}^{3}$.

As applied to $H$, our result can be compared with theorems stating that a complete convex hypersurface in Euclidean $n$-space $E^{n}$ splits into a product with a non-trivial linear factor if Euclidean mean curvature $H_{\epsilon} \equiv 1$ (see [7] and [3]) or if $1 \leq H_{\epsilon} \leq c$ for a certain constant $c<(n-1) /(n-2)$ (see [8]), or if $1 \leq H_{\epsilon} \leq$ $(n-1) /(n-2)$ with the upper limit $(n-1) /(n-2)$ sharp (see [9] and [10]). For related results involving spacelike or timelike convex hypersurfaces in Minkowski $n$-space $E_{1}^{n}$, see [11].

Received by the editors June 23, 1998.

1991 Mathematics Subject Classification. Primary 53C42, 53C40, 53B30.

(C)2000 American Mathematical Society 
Entire, spacelike surfaces in $E_{1}^{3}$ that are convex as surfaces in $E^{3}$ need not be cylindrical when $H \equiv$ constant $\neq 0$. (See [17].) This is not the only instance in which Euclidean results carry over to spacelike or timelike surfaces in $E_{1}^{3}$, but not to both. Bernstein's theorem holds in the spacelike case (see [2] and [4]) but fails in the timelike case (see [5] or [13).

It should also be noted that timelike surfaces in $E_{1}^{3}$ on which $H \equiv 0$ and $K \leq 0$ need not be cylindrical if they are complete as surfaces in $E^{3}$. (See [14] and [19].) One knows, however, that any such surface is conformally equivalent to $E_{1}^{2}$. (See [13.)

\section{Preliminaries}

Use the Minkowski scalar product $\langle$,$\rangle determined by the metric d s^{2}=d x^{2}+$ $d y^{2}-d z^{2}$ on $x, y, z$-space $R^{3}$ to define Minkowski 3 -space $E_{1}^{3}$. Similarly, use the Euclidean inner product $\langle,\rangle_{\epsilon}$ determined by the metric $d s^{2}=d x^{2}+d y^{2}+d z^{2}$ on $x, y, z$-space $R^{3}$ to define Euclidean 3 -space $E^{3}$. A surface $C^{2}$ immersed in $E_{1}^{3}$ is automatically $C^{2}$ immersed in $E^{3}$ as well (and vice versa). We work with the geometry induced on the surface by both ambient spaces. (For background, see 7.1-7.2 in [19.)

Suppose a surface $S$ is $C^{2}$ immersed in $E_{1}^{3}$. Call $S$ timelike (resp. spacelike) if all its tangent planes make a Euclidean angle of more than (resp. less than) $\pi / 4$ with the $x, y$-plane. Assume $S$ is timelike. Then, modulo a rigid motion of $E_{1}^{3}, S$ can be locally represented as the graph of a $C^{2}$ function $x=f(y, z)$ over a neighborhood $U$ in the $y, z$-plane. Thus $\varphi(y, z)=(f(y, z), y, z)$ is a $C^{2}$ imbedding $\varphi: U \rightarrow E_{1}^{3}$ with first fundamental form $I$, unit normal vector field $\nu$, second fundamental form $I I$, Gauss curvature $K$ and mean curvature $H$ given by

$$
\begin{gathered}
I=\langle d \varphi, d \varphi\rangle=\left(f_{y}{ }^{2}+1\right) d y^{2}+2 f_{y} f_{z} d y d z+\left(f_{z}{ }^{2}-1\right) d z^{2} \\
\nu=\left(1,-f_{y}, f_{z}\right) /\left(1+f_{y}{ }^{2}-f_{z}{ }^{2}\right)^{1 / 2} \\
I I=-\langle d \varphi, d \nu\rangle=\left(f_{y y} d y^{2}+2 f_{y z} d y d z+f_{z z} d z^{2}\right) /\left(1+f_{y}{ }^{2}-f_{z}{ }^{2}\right)^{1 / 2} \\
K=\operatorname{det} I I / \operatorname{det} I=\left(f_{y y} f_{z z}-f_{y z}{ }^{2}\right) /\left(1+f_{y}{ }^{2}-f_{z}{ }^{2}\right)^{2} \\
2 H=\operatorname{tr}_{I} I I=\left(\left(f_{y}{ }^{2}+1\right) f_{z z}-2 f_{y} f_{z} f_{y z}+\left(f_{z}{ }^{2}-1\right) f_{y y}\right) /\left(1+f_{y}{ }^{2}-f_{z}{ }^{2}\right)^{3 / 2},
\end{gathered}
$$

with $\langle\nu, \nu\rangle=1$ and

$$
\operatorname{det} I=-\left(1+f_{y}^{2}-f_{z}^{2}\right)<0,
$$

since $S$ is timelike. For the imbedding $\varphi: U \rightarrow E^{3}$, one gets the Euclidean first fundamental form $I_{\epsilon}$, unit normal vector field $\nu_{\epsilon}$, second fundamental form $I I_{\epsilon}$, Gauss curvature $K_{\epsilon}$ and mean curvature $H_{\epsilon}$ given by

$$
\begin{gathered}
I_{\epsilon}=\langle d \varphi, d \varphi\rangle_{\epsilon}=\left(f_{y}{ }^{2}+1\right) d y^{2}+2 f_{y} f_{z} d y d z+\left(f_{z}{ }^{2}+1\right) d z^{2}, \\
\nu_{\epsilon}=\left(1,-f_{y},-f_{z}\right) /\left(1+f_{y}{ }^{2}+f_{z}{ }^{2}\right)^{1 / 2} \\
I I_{\epsilon}=-\left\langle d \varphi, d \nu_{\epsilon}\right\rangle_{\epsilon}=\left(f_{y y} d y^{2}+2 f_{y z} d y d z+f_{z z} d z^{2}\right) /\left(1+f_{y}{ }^{2}+f_{z}{ }^{2}\right)^{1 / 2}, \\
K_{\epsilon}=\operatorname{det} I I_{\epsilon} / \operatorname{det} I_{\epsilon}=\left(f_{y y} f_{z z}-f_{y z}{ }^{2}\right) /\left(1+f_{y}{ }^{2}+f_{z}{ }^{2}\right)^{2}, \\
2 H_{\epsilon}=\operatorname{tr}_{I_{\epsilon}} I I_{\epsilon}=\left(\left(f_{y}{ }^{2}+1\right) f_{z z}-2 f_{y} f_{z} f_{y z}+\left(f_{z}{ }^{2}+1\right) f_{y y}\right) /\left(1+f_{y}{ }^{2}-f_{z}{ }^{2}\right)^{3 / 2} .
\end{gathered}
$$


Note that (11) and (2) give

$$
\operatorname{sign} K=-\operatorname{sign} K_{\epsilon}
$$

on a timelike surface $S$ that is $C^{2}$ immersed in $E_{1}^{3}$.

By a cylinder, we mean the set of all points on all lines perpendicular (in the Euclidean sense) to a plane $\Pi$ in $E^{3}$ that intersect a fixed curve $\gamma$ in $\Pi$. A surface $C^{2}$ immersed in $E^{3}$ with $I_{\epsilon}$ complete on which $K_{\epsilon} \equiv 0$ is a cylinder. (See [6].)

By a convex surface, we mean the boundary $\partial C$ of a closed, convex set $C$ in $E^{3}$ with non-empty interior. A surface that is $C^{2}$ immersed in $E^{3}$ with $I_{\epsilon}$ complete and $0 \not \equiv K_{\epsilon} \geq 0$ is actually imbedded and convex. (See [18] and [16].) Suppose now that $S$ is a timelike surface $C^{2}$ immersed in $E_{1}^{3}$. It follows from (3) that $S$ is convex and non-cylindrical as a surface in $E^{3}$ provided that $0 \not \equiv K \leq$ on $S$ and $S$ is $\mathbf{E}^{\mathbf{3}}$-complete (meaning that $I_{\epsilon}$ is complete on $S$ ).

A subset $T$ of the 2-sphere $S^{2}$ in $E^{3}$ is convex on $\mathbf{S}^{2}$ if and only if the shorter great circular arc joining any two non-antipodal points in $T$ lies in $T$, and at least one great circular arc joining any two antipodal points in $T$ lies in $T$. For any subset $T$ of $S^{2}$ (resp. on $R^{2}$ ), $\operatorname{Int}(T)$ denotes the interior of $T$ on $S^{2}$ (resp. on $R^{2}$ ). For any subset $T$ of $E^{3}, \operatorname{cl}(T)$ denotes the closure of $T$ in $E^{3}$. For an oriented surface $S$ that is $C^{2}$ immersed in $E^{3}$ (or in $E_{1}^{3}$ ), the Euclidean Gauss map $G_{\epsilon}: S \rightarrow S^{2}$ sends each point $p$ on $S$ to the point on $S^{2}$ described by the Euclidean unit normal vector $\nu_{\epsilon}$ to $S$ at $p$.

The following lemma summarizes known facts.

Lemma 1. Suppose a non-compact, oriented surface $S$ is $C^{2}$ imbedded in $E^{3}$ as the boundary $\partial C$ of a convex subset $C$ of $E^{3}$ with non-empty interior.

(i) If $C$ contains no lines, then $S$ is homeomorphic to $R^{2}$. (See [1].)

(ii) If $S$ is homeomorphic to $R^{2}$, there is a tangent plane $\Pi$ to $S$ such that for orthogonal projection $\pi: E^{3} \rightarrow \Pi$ with $C^{\prime}=\pi(C)$, the portion of $S$ in $\pi^{-1}\left(\operatorname{Int}\left(C^{\prime}\right)\right)$ is the graph of a $C^{2}$ function over $\operatorname{Int}\left(C^{\prime}\right)$. (See [20.)

(iii) If $C$ contains no lines, $\operatorname{cl}\left(G_{\epsilon}(S)\right)$ is convex on $S^{2}$. (See [20].)

Lemma 2. Suppose a non-compact, oriented surface $S$ is $C^{2}$ imbedded in $E^{3}$ as the boundary $\partial C$ of a convex subset $C$ of $E^{3}$ with non-empty interior. Let $P$ be a plane through the origin that intersects $\operatorname{Int}\left(G_{\epsilon}(S)\right) \neq \emptyset$. Let $\Sigma_{1}$ and $\Sigma_{2}$ be the intersections of $G_{\epsilon}(S)$ with the closed half spaces of $E^{3}$ bounded by $P$, and let $C^{\prime}=\pi(C)$ for orthogonal projection $\pi: E^{3} \rightarrow P$. Then

$$
\pi^{-1}\left(\operatorname{Int}\left(C^{\prime}\right)\right) \cap S=S_{1} \cup S_{2}
$$

where $S_{1}$ and $S_{2}$ are graphs of $C^{2}$ functions on $\operatorname{Int}\left(C^{\prime}\right) \neq \emptyset$, with $G_{\epsilon}\left(S_{1}\right) \subset \Sigma_{1}$ and $G_{\epsilon}\left(S_{2}\right) \subset \Sigma_{2}$.

Proof. Since $\operatorname{Int}\left(G_{\epsilon}(S)\right) \neq \emptyset$, the convex surface $S=\partial C$ is not a cylinder, and $C$ contains no lines. Thus $\pi^{-1}\left(p_{0}\right) \cap S \neq \emptyset$ for any $p_{0}$ in $\operatorname{Int}\left(C^{\prime}\right)$. Since the interior of the convex set $C$ in $E^{3}$ lies to one side of any tangent plane to $S=\partial C$, there are at most two points in $\pi^{-1}\left(p_{0}\right) \cap S$ for any $p_{0}$ in $\operatorname{Int}\left(C^{\prime}\right)$.

If $\pi^{-1}(p) \cap S$ contains exactly one point for a particular $p$ in $\operatorname{Int}\left(C^{\prime}\right)$, then $\pi^{-1}\left(p_{0}\right)$ contains exactly one point for every $p_{0}$ in $\operatorname{Int}\left(C^{\prime}\right)$. This follows since if $C$ contains the half line starting at $\pi^{-1}(p) \cap S$ in the direction of a vector $\xi$ perpendicular to $P$, then $C$ also contains the half line starting at any point in $\pi^{-1}\left(p_{0}\right) \cap S$ in the direction of $\xi$ for every $p_{0}$ in $\operatorname{Int}\left(C^{\prime}\right)$. 
But if $\pi^{-1}\left(p_{0}\right) \cap S$ contains exactly one point for every $p_{0}$ in $\operatorname{Int}\left(C^{\prime}\right)$, then $S_{0}=\pi^{-1}\left(\operatorname{Int}\left(C^{\prime}\right)\right)$ is the graph of a $C^{2}$ function over $\operatorname{Int}\left(C^{\prime}\right)$ in $P$, so that $G_{\epsilon}\left(S_{0}\right)$ lies in one of the two open hemispheres on $S^{2}$ bounded by $P$. This contradicts the assumption that $P$ intersects $\operatorname{Int}\left(G_{\epsilon}(S)\right)$, since $\nu_{\epsilon}$ lies on $P \cap S^{2}$ at any point on $\pi^{-1}\left(\partial C^{\prime}\right) \cap S$.

It follows that $\pi^{-1}\left(p_{0}\right) \cap S$ contains exactly two points for every $p_{0}$ in $S$. Thus $\pi^{-1}\left(\operatorname{Int}\left(C^{\prime}\right)\right) \cap S=S_{1} \cup S_{2}$, with $S_{1}$ and $S_{2}$ as described in the lemma.

\section{MAIN RESUltS}

The next lemma is at the heart of our arguments.

Lemma 3. Suppose that $S$ is a timelike surface $C^{2}$ immersed in $E_{1}^{3}$ with $K \leq 0$. Let $\alpha$ be the acute Euclidean angle between $\nu_{\epsilon}$ and the $x, y$-plane, so that $0 \leq \alpha<$ $\pi / 4$. Then

$$
K_{\epsilon}=-K \cos ^{2} 2 \alpha .
$$

If $S$ is also $E^{3}$ complete with $K \not \equiv 0$ (making $S$ convex as a surface in $E^{3}$ ), then

$$
\left|H_{\epsilon}\right| \geq|H| \cos ^{3 / 2} 2 \alpha \geq 0 \text {. }
$$

Proof. Let $p$ be a point on the timelike surface $S$. With no loss of generality, assume that a neighborhood $\mathcal{U}$ of $p$ on $S$ is the graph of a $C^{2}$ function $x=f(y, z)$ over an open set $U$ in the $y, z$-plane, with $p=\left(f\left(y_{0}, z_{0}\right), y_{0}, z_{0}\right)$. If $\beta$ is the angle between $\nu_{\epsilon}$ and the positive $z$-axis, (2) gives

$$
\cos ^{2} \beta=f_{z}^{2} /\left(1+f_{y}{ }^{2}+f_{z}{ }^{2}\right), \quad \cos ^{2} \alpha=\left(1+f_{y}{ }^{2}\right) /\left(1+f_{y}{ }^{2}+f_{z}{ }^{2}\right)
$$

since $\alpha= \pm\left(\frac{\pi}{2}-\beta\right)$, and

$$
\cos 2 \alpha=2 \cos ^{2} \alpha-1=\left(1+f_{y}{ }^{2}-f_{z}{ }^{2}\right) /\left(1+f_{y}{ }^{2}+f_{z}{ }^{2}\right) .
$$

Note that $2 \alpha$ is the angle between $\nu$ and $\nu_{\epsilon}$ at any point on $S$. By (1) and Lemma 3] one has

$$
K_{\epsilon}=\frac{\left(f_{y y} f_{z z}-f_{y z}{ }^{2}\right)}{\left(1+f_{y}{ }^{2}-f_{z}{ }^{2}\right)^{2}} \cdot \frac{\left(1+f_{y}{ }^{2}-f_{z}{ }^{2}\right)^{2}}{\left(1+f_{y}{ }^{2}+f_{z}{ }^{2}\right)^{2}}=-K \cos 2 \alpha
$$

with

$$
\begin{aligned}
2 H_{\epsilon}= & \frac{\left(\left(f_{y}{ }^{2}+1\right) f_{z z}-2 f_{y} f_{z} f_{y z}+\left(f_{z}{ }^{2}-1\right) f_{y y}\right)}{\left(1+f_{y}{ }^{2}-f_{z}{ }^{2}\right)^{3 / 2}} \cdot \frac{\left(1+f_{y}{ }^{2}-f_{z}{ }^{2}\right)^{3 / 2}}{\left(1+f_{y}{ }^{2}+f_{z}{ }^{2}\right)^{3 / 2}} \\
& +\frac{2 f_{y y}}{\left(1+f_{y}{ }^{2}+f_{z}{ }^{2}\right)^{3 / 2}}
\end{aligned}
$$

giving

$$
H_{\epsilon}=H \cos ^{3 / 2} 2 \alpha+\frac{f_{y y}}{\left(1+f_{y}^{2}+f_{z}^{2}\right)^{3 / 2}} .
$$

Note that (5) establishes the first claim in the lemma, since the choice of $p$ was arbitrary.

Suppose now that $S$ is also $E^{3}$ complete with $K \not \equiv 0$, so that $S$ is convex as a surface in $E^{3}$. Then $\mathcal{U}$ is concave up or concave down with respect to the positive $x$-axis. With no loss of generality, assume the $\mathcal{U}$ is concave up, so that $f_{y y} \geq 0$, $f_{z z} \geq 0$ and $H_{\epsilon} \geq 0$ on $U$. 
Let $\gamma$ be the connected curve in $\mathcal{U} \cap\left\{z=z_{0}\right\}$ given by $\gamma=\gamma(y)=\left(f\left(y, z_{0}\right), y, z_{0}\right)$ with $p=\gamma\left(y_{0}\right)$. The curvature of $\gamma(y)$ as a curve in $E^{3}$ is

$$
k(\gamma(y))=\frac{f_{y y}}{\left(1+f_{y}{ }^{2}\right)^{3 / 2}} \geq 0,
$$

and the principal unit normal vector to $\gamma(y)$ is

$$
\mathbf{N}=\mathbf{N}(y)=\left(1,-f_{y}, 0\right) /\left(1+f_{y}{ }^{2}\right)^{1 / 2} .
$$

If $\theta$ is the angle between $\nu_{\epsilon}$ and $\mathbf{N}(y)$, then

$$
\begin{aligned}
\cos \theta=\left\langle\mathbf{N}, \nu_{\epsilon}\right\rangle_{\epsilon} & =\left\langle\left(1,-f_{y}, 0\right),\left(1,-f_{y},-f_{z}\right)\right\rangle_{\epsilon} /\left(1+f_{y}{ }^{2}\right)^{1 / 2}\left(1+f_{y}{ }^{2}+f_{z}{ }^{2}\right)^{1 / 2} \\
& =\left(1+f_{y}{ }^{2}\right)^{1 / 2}\left(1+f_{y}{ }^{2}+f_{z}{ }^{2}\right)^{1 / 2} \geq 0 .
\end{aligned}
$$

Thus (7) and (8) give

$$
\begin{aligned}
\frac{f_{y y}}{\left(1+f_{y}{ }^{2}+f_{z}{ }^{2}\right)^{3 / 2}} & =\frac{f_{y y}}{\left(1+f_{y}{ }^{2}\right)^{3 / 2}} \cdot \frac{\left(1+f_{y}{ }^{2}\right)^{3 / 2}}{\left(1+f_{y}{ }^{2}+f_{z}{ }^{2}\right)^{3 / 2}} \\
& =k(\gamma(y)) \cos ^{3}(\theta) \geq 0 .
\end{aligned}
$$

By Meusnier's theorem,

$$
k(\gamma(y)) \cos \theta=k_{n}\left(\gamma^{\prime}(y)\right) \geq 0,
$$

where $k_{n}\left(\gamma^{\prime}(y)\right)$ is the normal curvature of $S$ at $\gamma(y)$ in the $\gamma^{\prime}(y)$ direction. Thus (9) becomes

$$
\frac{f_{y y}}{\left(1+f_{y}^{2}+f_{z}^{2}\right)^{3 / 2}}=k_{n}\left(\gamma^{\prime}(y)\right) \cos ^{2} \theta \geq 0
$$

with (6) giving

$$
H_{\epsilon}=H \cos ^{3 / 2} 2 \alpha+k_{n}\left(\gamma^{\prime}(y)\right) \cos ^{2} \theta \geq 0 .
$$

If $H \geq 0$, then (10) and (11) yield

$$
\left|H_{\epsilon}\right| \geq|H| \cos ^{3 / 2} 2 \alpha .
$$

If $H \leq 0$, then (10) and (11) yield

$$
|H| \cos ^{3 / 2} 2 \alpha=-H \cos ^{3 / 2} 2 \alpha=k_{n}\left(\gamma^{\prime}(y)\right) \cos ^{2} \theta-H_{\epsilon} .
$$

But $2 H_{\epsilon}$ is the sum of normal curvatures in orthogonal tangent directions at any point on $S$. Thus at any point on $\gamma(y)$,

$$
2 H_{\epsilon}=k_{n}\left(\gamma^{\prime}(y)\right)+k_{n}(\mathbf{w}(y))
$$

for $\mathbf{w}(y)=\nu_{\epsilon}(\gamma(y)) \times \gamma^{\prime}(y)$, with both terms non-negative on the right side of (14). When $H \leq 0$, (13) and (14) give

$$
\begin{aligned}
& 2|H| \cos ^{3 / 2} 2 \alpha=2 k_{n}\left(\gamma^{\prime}(y)\right) \cos ^{2} \theta-2 H_{\epsilon} \leq 2 k_{n}\left(\gamma^{\prime}(y)\right)-2 H_{\epsilon} \\
= & k_{n}\left(\gamma^{\prime}(y)\right)-k_{n}(\mathbf{w}(y)) \leq k_{n}\left(\gamma^{\prime}(y)\right)+k_{n}(\mathbf{w}(y))=2 H_{\epsilon}=2\left|H_{\epsilon}\right| .
\end{aligned}
$$

This shows that (12) holds at any point on $\gamma(y)$, and hence at $p$. Since $p$ was arbitrary, the second claim in the lemma is established. 
In the remainder of this paper, let $\Pi^{+}$and $\Pi^{-}$denote the planes $z=\sqrt{2} / 2$ and $z=-\sqrt{2} / 2$ respectively, with

$$
\begin{aligned}
\Sigma & =S^{2} \cap\{-\sqrt{2} / 2 \leq z \leq \sqrt{2} / 2\}, \\
\Sigma^{+} & =S^{2} \cap \Pi^{+}, \quad \Sigma^{-}=S^{2} \cap \Pi^{-},
\end{aligned}
$$

and

$$
\begin{gathered}
R=\Sigma \cap\{x \geq 0\}, R^{+}=R \cap \Pi^{+}, R^{-}=R \cap \Pi^{-}, \\
R_{0}^{+}=R^{+} \cap\{x=0\}, R_{0}^{-}=R^{-} \cap\{x=0\} .
\end{gathered}
$$

Suppose $p$ lies in $R_{0}^{+}$, so its antipodal point $q$ lies in $R_{0}^{-}$. There is a unique plane $\Pi(p, q)$ in $E^{3}$ containing the parallel lines tangent to $\Sigma^{+}$at $p$ and $\Sigma^{-}$at $q$. Moreover,

$$
\Gamma_{p, q}=\Pi(p, q) \cap S^{2}
$$

is the only great circle on $S^{2}$ through $p$ and $q$ that is contained in $\Sigma$.

Lemma 4. Suppose that $\Omega \subset R$ with $\operatorname{cl}(\Omega)$ convex on $S^{2}$ and $\operatorname{Int}(\Omega) \neq \emptyset$. Let $X=\operatorname{cl}(\Omega) \cap\left\{\Pi^{+} \cup \Pi^{-}\right\}$. Then there is a plane $P$ through the origin intersecting Int $(\Omega)$ that bounds an open half space of $E^{3}$ containing $X$, and contains no pair of antipodal points from $\mathrm{cl}(\Omega)$.

Proof. Because $\operatorname{cl}(\Omega)$ is convex on $S^{2}, \operatorname{cl}(\Omega) \cap \Pi^{+}$and $\operatorname{cl}(\Omega) \cap \Pi^{-}$each contains at most one point, so that $X$ contains at most two points. Suppose that $X$ contains two points, $p$ in $\operatorname{cl}(\Omega) \cap \Pi^{+}$and $q$ in $\operatorname{cl}(\Omega) \cap \Pi^{-}$. We claim that $p$ and $q$ are not antipodal. Otherwise, $x=0$ at $p$ and $q$ since $\operatorname{cl}(\Omega) \subset \operatorname{cl}(R) \subset\{x \geq 0\}$. Thus $p$ lies in $R_{0}^{+}, q$ lies in $R_{0}^{-}$and $\Gamma_{p, q}=\Pi(p, q) \cap S^{2}$ is the only great circle on $S^{2}$ through $p$ and $q$ contained in $\Sigma$. Since $\operatorname{Int}(\Omega) \neq \emptyset$, there is a point $m$ in $\operatorname{Int}(\Omega)$ that does not lie on the plane $\Pi(p, q)$. Thus the great circle $\tilde{\Gamma}_{p, q}$ through $p, q$ and $m$ is not $\Gamma_{p, q}$, and is not contained in $\Sigma$. But the convexity of $c l(\Omega)$ on $S^{2}$ guarantees that the semicircle $\tilde{\Gamma}_{p, q}^{R}=\tilde{\Gamma}_{p, q} \cap\{x \geq 0\}$ is contained in $\operatorname{cl}(\Omega) \subset \Sigma$, since $p, q$ and $m$ all lie on $\tilde{\Gamma}_{p, q}^{R} \cap \operatorname{cl}(\Omega)$. If $\tilde{\Gamma}_{p, q}^{R}$ lies in $\Sigma$, so does $\tilde{\Gamma}_{p, q}$. This contradiction confirms that $p$ and $q$ are not antipodal.

Let $\gamma_{1}$ be the shorter great circular arc joining $p$ to $q$ in $\operatorname{cl}(\Omega)$, and let $P_{1}$ be the plane containing $\gamma_{1}$. The line $l_{p, q}$ through $p$ and $q$ does not pass through the origin, since $p$ and $q$ are not antipodal. Since $\operatorname{Int}(\Omega) \neq \emptyset$, there is a point $m$ in $\operatorname{Int}(\Omega)$ which lies off $P_{1}$. Let $P$ be the plane through $m$ containing the line $l$ through the origin that is parallel to $l_{p, q}$. No point of $l_{p, q}$ lies on $P$, since otherwise the contradiction $P=P_{1}$ would follow. Thus $X=\{p, q\}$ is contained in one of the open half spaces in $E^{3}$ bounded by $P$. Finally, since $P_{1} \cap \operatorname{cl}(\Omega)=\gamma_{1}$ contains no pair of antipodal points, and since $P$ converges to $P_{1}$ as $m$ approaches $\gamma_{1}$ in $\operatorname{Int}(\Omega)$, we can choose $m$ so close to $\gamma_{1}$ in $\operatorname{Int}(\Omega)$ that $P \cap \operatorname{cl}(\Omega)$ contains no antipodal points.

The cases in which at least one of the sets $\operatorname{cl}(\Omega) \cap \Pi^{+}$or $\operatorname{cl}(\Omega) \cap \Pi^{-}$is empty can be handled in a similar way, but with easier arguments.

Theorem. Suppose that $S$ is an $E^{3}$ complete timelike surface $C^{2}$ immersed in $E^{3}$ with $0 \not \equiv K<0$ (so that $S$ is convex as a surface in $E^{3}$ ). Then neither $H$ nor $K$ can be bounded away from zero on $S$.

Proof. Since $K \not \equiv 0$ on $S, K_{\epsilon} \not \equiv 0$ by (3), so that $S$ is not a cylinder. This implies that the closed convex set $C$ in $E^{3}$ with $S=\partial C$ contains no lines. By Lemma प(i),

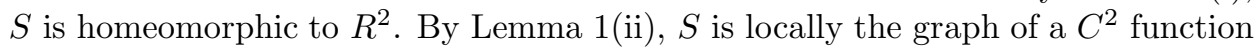


over one of its tangent planes, which we assume, with no loss of generality, to be the $y, z$-plane, with $S$ lying in the half space $\{x \geq 0\}$. Then $G_{\epsilon}(S) \subset R$ for the subset $R$ of $S^{2}$ defined in (16).

By Lemma प(iii), $c l\left(G_{\epsilon}(S)\right)$ is a convex subset of $S^{2}$. Thus Lemma 4 applies with $\Omega=G_{\epsilon}(S)$, giving a plane $P$ through the origin with $P \cap \operatorname{Int}\left(G_{\epsilon}(S)\right) \neq \emptyset$, with

$$
X=\operatorname{cl}\left(G_{\epsilon}(S)\right) \cap\left\{\Pi^{+} \cup \Pi^{-}\right\}
$$

contained in one of the open half spaces $H_{1}$ or $H_{2}$ in $E^{3}$ bounded by $P$, and with $P \cap \operatorname{cl}\left(G_{\epsilon}(S)\right)$ containing no pair of antipodal points. Index $H_{1}$ and $H_{2}$ so that $X$ lies in $H_{2}$. Then if $G_{1}=G_{\epsilon}(S) \cap \operatorname{cl}\left(H_{1}\right)$ and $G_{2}=G_{\epsilon}(S) \cap \operatorname{cl}\left(H_{2}\right)$, one has $X \cap G_{1}=\emptyset$. Apply Lemma 2 with $\pi: E^{3} \rightarrow P$ orthogonal projection onto $P$ and $C^{\prime}=\pi(C)$, so that $\pi^{-1}\left(\operatorname{Int}\left(C^{\prime}\right)\right) \cap S=S_{1} \cup S_{2}$, with $S_{1}$ and $S_{2}$ each the graph of a $C^{2}$ function on $\operatorname{Int}\left(C^{\prime}\right)$. Index $S_{1}$ and $S_{2}$ so that $G_{\epsilon}\left(S_{1}\right) \subset G_{1}$.

We claim that $C^{\prime}$ cannot be contained between parallel lines $l_{1}$ and $l_{2}$ in $P$. Otherwise, $S=\partial C$ would be contained in the closed region bounded by the planes $P_{1}$ and $P_{2}$ through $l_{1}$ and $l_{2}$ respectively that are perpendicular to $P$. Since $C$ is convex, $\operatorname{cl}\left(G_{\epsilon}(S)\right)$ would contain antipodal points perpendicular to $P_{1}$ and $P_{2}$, and lying in $P$. This would contradict the fact that $P \cap \operatorname{cl}\left(G_{\epsilon}(S)\right)$ contains no antipodal points, so our claim is verified.

Since $S \subset\{x \geq 0\}$ is a graph over the $y, z$-plane, $C$ contains a half line that is not perpendicular to $P$. Thus $\operatorname{Int}\left(C^{\prime}\right)$ contains a half line $\lambda$. Since the convex set $C^{\prime}$ in $P$ is not contained between any two lines parallel to $\lambda$, one easily argues that for any $r>0, \operatorname{Int}\left(C^{\prime}\right)$ contains a disk of radius $r$. Let $D$ be a disk of radius $2 r$ contained in $\operatorname{Int}\left(C^{\prime}\right)$ and centered at a point $c$. Let $l$ be the line through $c$ perpendicular to $P$. Take a closed ball $B$ of radius $r$ centered on $l$, starting on the same side of $S_{1}$ as $C$, with $B \cap S_{1}=\emptyset$. Move $B$ toward $S_{1}$ keeping its center on $l$ until it first touches $S_{1} \cap \pi^{-1}(D)$ at a point $p$. All of $B$ now lies on $S_{1}$ or to the same side of $S_{1}$ as $C$. Thus $0 \leq\left|H_{\epsilon}(p)\right| \leq 1 / r$ and $K_{\epsilon}(p) \leq 1 / r^{2}$. On the other hand, we took $G_{\epsilon}\left(S_{1}\right) \subset G_{1}$ with $X \cap G_{1}=\emptyset$. Thus there is a constant $\alpha_{0}$ satisfying $0 \leq \alpha \leq \alpha_{0}<\pi / 4$, where $\alpha$ is the angle $\nu_{\epsilon}$ on $S_{1}$ makes with the $x, y$-plane. By Lemma 3 , the inequalities

$$
\left|H_{\epsilon}\right| \geq|H| \cos ^{3 / 2} 2 \alpha_{0} \geq 0, \quad K_{\epsilon} \geq|K| \cos ^{2} 2 \alpha_{0}
$$

hold on $S_{1}$. If $H$ or $K$ is bounded away from zero on $S$, we obtain a contradiction to (17) at $p$ by taking $r$ sufficiently large. This establishes the theorem.

Corollary. If $H \equiv$ constant $\neq 0$ and $K \leq 0$ on an $E^{3}$ complete timelike surface $C^{2}$ immersed in $E_{1}^{3}$, then (up to rigid motions of $E_{1}^{2}$ ) $S$ is a cylinder over a circle of radius $r=1 / 2 \mathrm{H}$ in the $x, y$-plane, or over a hyperbola of curvature $2 \mathrm{H}$ in the $y, z$-plane.

Proof. By our theorem, $K \equiv 0$ must hold on $S$, making $K_{\epsilon} \equiv 0$ and $S$ a cylinder. The corollary then follows by elementary arguments.

\section{ACKNOWLEDGEMENT}

The author wishes to thank Tilla Weinstein for suggestions which improved the exposition of this paper. 


\section{REFERENCES}

[1] Busemann H, Convex Surfaces, Interscience, New York, (1958). MR 21:3900

[2] Calabi E., Examples of Bernstein problems for some non-linear equations, Proc. Sympos. Pure Appl. Math. 15(1968),223-230. MR 41:8806

[3] Cheng S.Y. and Yau S.T., Differential equations on Riemannian manifolds and their geometrical applications, Comm. Pure. Appl. Math., 28(1975),333-354. MR 52:6608

[4] - Maximal spacelike hypersurfaces in the Lorentz-Minkowski spaces, Ann. of Math. (2)104(1976), 407-419

[5] Flaherty F.J., Surfaces d'onde dans l'espace de Lorentz-Minkowski, C.R. Acad. Sci. Paris t. 284A, 521-523. MR 55:4064

[6] Hartman P. and Nirenberg L., On spherical image maps whose Jacobians do not change sign, Amer. J. Math., 81(1959),901-920. MR 23:A4106

[7] Klotz T. and Osserman R., Complete surfaces in $E^{3}$ with constant mean curvature, Comm. Math. Helv., 41(1966-67),313-318. MR 35:2213

[8] Kohlmann P., Splitting and pinching for convex sets, Geom. Dedicata, 60(1996),125-143. MR 97a:52005

[9] Lin S., Splitting and pinching for complete convex surfaces in $E^{3}$, Geom. Dedicata 69(1998), 83-90. MR. 98k:53006

[10] A mean cuvature pinching theorem for convex hypersurfaces in $E^{n+1}$. Preprint.

[11] $\longrightarrow$, Spacelike and timelike convex hypersurfaces in Minkowski space with $H \geq c>0$, Preprint.

[12] Milnor T.K., Harmonic maps and classical surface theory in Minkowski 3-space, Trans. Amer. Math. Soc. 280, 1983,161-185. MR 85e:58037

[13] Milnor T.K., A conformal analog of Bernstein's theorem for timelike surfaces in Minkowski 3-space, Contemp. Math. 64(1987),123-130. MR 88b:53076

[14] Milnor T.K., Entire timelike minimal surfaces in $E^{3,1}$, Mich. Math. J., 37(1990),163-177. MR 92b:53007

[15] O'Neill B,. Semi-Riemannian Geometry with Applications to Relativity, Academic Press (1983).

[16] Sacksteder R., On hypersurfaces with nonnegative sectional curvature, Amer. J. Math.,82(1960),609-630. MR 22:7087

[17] Treibergs A.E., Entire spacelike hyperspaces of constant mean curvature in Minkowski space, Invent. Math. 66(1982),39-56. MR 83h:53080

[18] van Heijenoort J., On locally convex manifolds, Comm. Pure Appl. Math.,5(1952),223-242. MR 14:557b

[19] T.Weinstein, An Introduction to Lorentz Surfaces, de Gruyter Expositions in Mathematics 22 (1996). MR 98a:53104

[20] Wu H., The spherical images of convex hypersurfaces, J. Diff. Geom., 9(1974),279-290. MR 50:1182

Department of Mathematics, Rutgers University, New Brunswick, New Jersey 08903

E-mail address: senchun@math.rutgers.edu 